\title{
Management and prognosis of patients with liver metastases from gestational trophoblastic neoplasia: a retrospective cohort study
}

This article was published in the following Dove Press journal: Cancer Management and Research

\section{Liju Zong \\ Junjun Yang \\ Xiaoyu Wang \\ Yujia Kong \\ Tong Ren \\ Jun Zhao \\ Fengzhi Feng \\ Xirun Wan \\ Yang Xiang}

Departments of Obstetrics and Gynecology, Peking Union Medical College Hospital, Chinese Academy of Medical Sciences and Peking Union Medical College, Beijing, People's Republic of China
Correspondence: Junjun Yang Department of Obstetrics and Gynecology, Peking Union Medical College Hospital, Chinese Academy of Medical Sciences and Peking Union Medical College, No.I Shuaifuyuan, Wangfujing, Dongcheng District, Beijing 100730, People's Republic of China $\mathrm{Tel} / \mathrm{fax}+861065296068$ Email yangjunjun@pumch.cn
Purpose: The aims of this study were to analyze the clinical features, identify prognostic factors, and evaluate the survival outcomes of gestational trophoblastic neoplasia (GTN) patients with liver metastases.

Patients and methods: Forty patients with liver metastases arising from GTNs, who were treated at the Peking Union Medical College Hospital (Beijing, People's Republic of China) between January 1999 and December 2015, were recruited from the institutional database, and their medical records were reviewed. Univariate and multivariate analyses were performed to identify independent risk factors for survival.

Results: Twenty-seven patients $(67.5 \%)$ achieved complete remission after multidrug chemotherapy treatment. The remaining 13 patients $(32.5 \%)$ had disease progression. Eighteen patients $(45.0 \%)$ died during treatment or follow-up. A history of failed multidrug chemotherapy was an independent risk factor for survival (OR: 5.57, 95\% CI: 1.42-21.86, $P=0.014$ ). Moreover, patients with an International Federation of Gynecology and Obstetrics (FIGO) score of $>16$ had a significantly poorer survival than those with a score of $\leq 16(P<0.001)$.

Conclusion: GTN with liver metastasis is a very rare disease with a relatively poor prognosis. Patients with a history of failed multidrug chemotherapy and a FIGO score of $>16$ have poorer survival outcomes. Multidrug chemotherapy is the key to the management of GTN patients with liver metastases.

Keywords: multidrug chemotherapy failure, FIGO score, multidrug chemotherapy, hepatic metastasis

\section{Introduction}

Gestational trophoblastic neoplasia (GTN) refers to a group of uncommon gynecological malignancies, including choriocarcinoma, placental site trophoblastic tumor (PSTT), and epithelioid trophoblastic tumor (ETT). ${ }^{1}$ Owing to the ability to precisely measure beta-human chorionic gonadotropin ( $\beta$-hCG) levels and the disease's sensitivity to chemotherapy, the cure rates for GTNs are reportedly as high as $90 \%$, even in patients with widespread metastases. ${ }^{1}$ However, the prognosis of certain GTN patients is still poor. ${ }^{2,3}$ Furthermore, liver metastasis has been regarded as an independent risk factor of prognosis in previous reports.,

Liver metastasis is reported to occur in $1.8 \%-7.7 \%$ of patients with $\mathrm{GTN}^{6}$ and in $19.0 \%$ of patients with Stage IV GTN. ${ }^{7}$ However, the survival rates of patients with liver metastases have been reported to be as low as $10 \%-55 \%$. ${ }^{3,6,8-10}$ Considering the low incidence and poor prognosis of patients with liver metastases from GTN, we conducted a retrospective analysis of GTN patients with liver metastases who were treated at the Peking 
Union Medical College Hospital (PUMCH) (Beijing, People's Republic of China). The aims of this study were to analyze the clinical features, identify prognostic factors, and evaluate the survival outcomes of GTN patients with liver metastases.

\section{Patients and methods Data collection}

For this study, 2,067 patients with GTN were treated at PUMCH between January 1999 and December 2015; 40 patients with liver metastases (1.9\%) were diagnosed during this period. Patients were identified through a review of the institutional database. All clinical data were anonymized. This study was approved by the Ethics Committee of PUMCH, Beijing, People's Republic of China. Owing to the retrospective study design and analysis of clinical data, written informed consent was formally waived by the Ethics Committee of PUMCH. Patients were excluded from the study if they were diagnosed with pathologically confirmed PSTT and ETT because these diseases have distinct behaviors. The diagnosis of liver metastases from GTN was made based on previously confirmed GTN combined with the presence of new liver lesions discovered by ultrasonography, computed tomography, or magnetic resonance imaging. The diagnosis of 2 patients was pathologically confirmed by hepatic lesion biopsy or resection at another hospital prior to admission to PUMCH. Among the 40 GTN patients with liver metastases, $20(50.0 \%)$ were defined as ultra-high-risk GTN patients. This patient population has been reported previously. ${ }^{11}$

\section{Pretreatment evaluation}

All 40 GTN patients with liver metastases underwent pretreatment evaluation, which included a recording of the complete medical history, clinical examinations, determination of complete blood counts, as well as performance of coagulation tests, liver and renal function tests, serum $\beta$-hCG measurements, transvaginal or transabdominal ultrasonography, chest radiography, and abdominopelvic computed tomography or magnetic resonance imaging.

\section{Treatment assessment}

During treatment, serum $\beta$-hCG levels, complete blood counts, and liver and renal function proteins were tested weekly to evaluate therapeutic efficacy and monitor toxicity. Complete remission (CR) referred to normal serum $\beta$-hCG levels measured for 3 consecutive weeks. Relapse was defined as increased serum $\beta$-hCG 1 month after CR. Disease progression (PD) was defined as elevated or plateaued serum
$\beta$-hCG levels or the appearance of new metastases after $\geq 2$ consecutive courses of chemotherapy.

\section{Statistical analyses}

Variables in the univariate analysis were classified into qualitative and quantitative indicators before analysis. In the univariate analysis, qualitative variables were examined using Fisher's exact test, and quantitative variables were examined using Student's $t$-test or the Wilcoxon rank-sum test. Multivariate analysis was performed using a cumulative logistic regression model. Survival curves were plotted using the Kaplan-Meier method and compared by the log-rank test. To determine the optimal cutoff point of International Federation of Gynecology and Obstetrics (FIGO) scores that best predict the prognosis of GTN with liver metastases, the sensitivity and specificity were calculated using receiver operating characteristic (ROC) curve analysis. Youden's index was calculated as $J=$ sensitivity + specificity -1 . All statistical analyses were conducted using the SPSS for Windows, software version 20.0 (IBM Corporation, Armonk, NY, USA). A value of $P<0.05$ was considered statistically significant.

\section{Results}

\section{Clinical characteristics}

The median age of the patients was 33 (range: $21-54$ ) years. The median gravidity was 3 (range: 1-6), and the median parity was 1 (range: $0-4)$. Ten patients $(25.0 \%)$ had a molar antecedent pregnancy, $14(35.0 \%)$ had a nonmolar abortion, and $16(40.0 \%)$ had a full-term pregnancy. The interval from the index pregnancy was $>12$ months in 28 patients $(70.0 \%)$. Pretreatment serum $\beta$-hCG levels ranged from 39.2 to $3.3 \times$ $10^{6} \mathrm{IU} / \mathrm{L}$ before receiving treatment at $\mathrm{PUMCH}$. According to the FIGO 2000 scoring system for GTN, ${ }^{12}$ the median FIGO score was 16 (range: 7-22). Thirty-three patients $(82.5 \%)$ were defined as ultra-high-risk patients, with FIGO score $\geq 12$ based on the FIGO Cancer Report 2015 . $^{1}$

All GTN patients with liver metastases also had lung metastases. Distant metastatic sites (excluding the liver and lung) included the brain $(\mathrm{n}=12 ; 30.0 \%)$, kidneys $(\mathrm{n}=5$; $12.5 \%)$, and spleen $(n=4 ; 10.0 \%)$. Other rare sites included the gastrointestinal tract, adrenal gland, and pancreas. Besides lung metastases, 20 patients $(50.0 \%)$ presented with isolated liver metastases; 14 patients $(35.0 \%)$ displayed doublesite distant metastases, and 6 patients $(15.0 \%)$ presented with triple-site distant metastases. In addition, 20 patients $(50.0 \%)$ had a history of failed multidrug chemotherapy, exhibiting multidrug resistance or unsatisfactory reductions 
in serum $\beta$-hCG levels after treatment at other institutions. Furthermore, 6 patients underwent surgery (hysterectomy $[\mathrm{n}=2]$, partial resection of the intestine $[\mathrm{n}=1]$, hepatic lesion resection $[\mathrm{n}=2]$, and craniotomy $[\mathrm{n}=1]$ ) prior to admission to PUMCH.

Detailed clinical characteristics of the patients are summarized in Table 1.

\section{Chemotherapy}

Of the 40 GTN patients with liver metastases, 3 (7.5\%) died during their first course of chemotherapy (gastrointestinal hemorrhage [ $\mathrm{n}=1]$, brain herniation $[\mathrm{n}=1]$, and multiple organ failure $[n=1])$. In total, 37 patients $(92.5 \%)$ received multidrug chemotherapy at PUMCH. Of whom, 35 (94.6\%) received floxuridine, actinomycin-D, etoposide, and vincristine (FAEV), and 2 (5.4\%) received etoposide, methotrexate, and actinomycin-D/cyclophosphamide and vincristine (EMA/CO). The details of the FAEV regimen have been described in our previous report. ${ }^{13}$ The 2 patients who were initially treated with EMA/CO exhibited increasing or plateauing serum $\beta$-hCG levels and were treated with FAEV as

Table I Characteristics of gestational trophoblastic neoplasia patients with liver metastasis

\begin{tabular}{|c|c|c|c|}
\hline Characteristics & & $\mathbf{N}=\mathbf{4 0}$ & $\%$ \\
\hline \multirow[t]{2}{*}{ Age, years } & $<40$ & 32 & 80.0 \\
\hline & $\geq 40$ & 8 & 20.0 \\
\hline Interval from index & $<4$ & 8 & 20.0 \\
\hline \multirow[t]{3}{*}{ pregnancy, months } & $4-6$ & 3 & 7.5 \\
\hline & $7-12$ & I & 2.5 \\
\hline & $>12$ & 28 & 70.0 \\
\hline Pretreatment $\beta$-hCG, & $<10^{3}$ & 3 & 7.5 \\
\hline \multirow[t]{3}{*}{$\mathrm{IU} / \mathrm{L}$} & $10^{3}-10^{4}$ & 7 & 17.5 \\
\hline & $10^{4}-10^{5}$ & 13 & 32.5 \\
\hline & $>10^{5}$ & 17 & 42.5 \\
\hline Previous failed & Yes & 20 & 50.0 \\
\hline chemotherapy & No & 20 & 50.0 \\
\hline Site of metastases & Liver & 20 & 50.0 \\
\hline \multirow[t]{6}{*}{ (lung excluded)* } & Liver + brain & 8 & 20.0 \\
\hline & Liver + brain + kidney & 2 & 5.0 \\
\hline & Liver + brain + spleen & 2 & 5.0 \\
\hline & Liver + kidney & 2 & 5.0 \\
\hline & Liver + gastrointestinal tract & 2 & 5.0 \\
\hline & Liver + other sites** & 4 & 10.0 \\
\hline Number of & I & 20 & 50.0 \\
\hline metastatic sites (lung & 2 & 14 & 35.0 \\
\hline excluded) & 3 & 6 & 15.0 \\
\hline \multirow[t]{2}{*}{ FIGO score } & $7-11$ & 7 & 17.5 \\
\hline & $\geq 12$ & 33 & 82.5 \\
\hline
\end{tabular}

Notes: *All patients presented lung metastasis; **including the spleen, pancreas, and adrenal glands.

Abbreviations: $\beta$-hCG, $\beta$-human chorionic gonadotropin; FIGO, the International Federation of Gynecology and Obstetrics. a salvage treatment. Of the 35 patients who were initially treated with FAEV, 19 received EMA/CO $(n=8)$ along with EMA/etoposide and cisplatin (EP) $(n=6$; EMA/CO + EMA/ $\mathrm{EP})$ or paclitaxel and etoposide (TE)/paclitaxel and cisplatin (TP) $(\mathrm{n}=5 ; \mathrm{EMA} / \mathrm{CO}+\mathrm{TE} / \mathrm{TP})$ as salvage therapy (Table 2$)$.

In addition to systemic chemotherapy, GTN patients with brain metastases received intrathecal injection of methotrexate. Further 2-4 courses of consolidation chemotherapy were administered to the patients when the serum $\beta$-hCG levels were normal.

\section{Surgical intervention}

Surgery was performed in 14 patients $(35.0 \%)$, including pelvic operations (hysterectomy or uterine lesion resection; $\mathrm{n}=8$ ), thoracic surgeries (pulmonary lobectomy or lesion resection; $n=6)$, liver lesion resection $(n=1)$, and resection of a unilateral adrenal metastasis $(n=1)$. The aim of adjuvant surgery in these 14 patients was to remove the isolated drug-resistant tumor.

\section{Outcomes}

Of the 37 patients who survived the first course of chemotherapy, 27 (73.0\%) achieved CR after comprehensive treatment, and $10(27.0 \%)$ exhibited progressive disease. The median follow-up for the CR patients was 57 (range: 16-156) months. Five (18.5\%) of the 27 CR patients relapsed at 4-24 months after completion of treatment. Four (80.0\%) of the relapsed patients received salvage chemotherapy with FAEV or EMA/CO. However, all 4 patients progressed and died of complications. The other relapsed patient achieved CR after 6 courses of EMA/CO and 3 courses of TE. However, 6 months later, this patient relapsed again and died of intracranial hemorrhage.

In total, 18 patients died during the treatment and followup period, yielding an overall death rate of $45.0 \%$. Thirteen patients $(72.2 \%)$ had a history of failed chemotherapy prior

Table 2 Chemotherapy regimens in gestational trophoblastic neoplasia patients with liver metastases $(n=37)$

\begin{tabular}{lll}
\hline Initial treatment & Salvage regimen & Patients, $\mathbf{n}$ (\%) \\
\hline EMA/CO & FAEV & $2(5.4)$ \\
FAEV & EMA/CO + EMA/EP & $6(16.2)$ \\
FAEV & EMA/CO & $8(21.6)$ \\
FAEV & EMA/CO + TE/TP & $5(13.5)$ \\
FAEV & No salvage regimen & $16(43.2)$ \\
\hline
\end{tabular}

Abbreviations: CO, cyclophosphamide and vincristine; EMA, etoposide, methotrexate, actinomycin-D; EP, etoposide and cisplatin; FAEV, floxuridine, actinomycin-D, etoposide, and vincristine; TE, paclitaxel and etoposide; TP, paclitaxel and cisplatin. 
to their admission to $\mathrm{PUMCH}$. The remaining 5 patients $(27.8 \%)$ received initial treatment at $\mathrm{PUMCH}$.

\section{Prognostic factors}

Potential prognostic variables were classified into qualitative and quantitative indicators (Tables 3 and 4). Univariate analysis of these factors revealed that an antecedent term pregnancy $(P=0.013)$, a history of failed multidrug chemotherapy $(P=0.011)$, and a FIGO score $\geq 12 \quad(P=0.002)$ negatively influenced the prognosis of GTN patients with liver metastases. Although brain and renal metastases were not significantly associated with patient prognosis, 5 (41.7\%) of 12 patients with brain metastases and $2(40.0 \%)$ of 5 patients with renal metastases died of their disease.
Multivariate analysis (excluding FIGO scores) revealed that a history of failed multidrug chemotherapy was an independent risk factor for survival (OR: 5.57; 95\% CI: $1.42-21.86 ; P=0.014)$.

\section{Optimal cutoff point of FIGO score}

The sensitivity, specificity, and Youden's index of various FIGO scores were calculated to determine the optimal cutoff point for predicting prognosis. FIGO score of $>16$ had the maximum Youden's index (0.537), a sensitivity of $63.2 \%$, and a specificity of $90.5 \%$ for predicting death from GTN. This suggests that patients with a FIGO score of $>16$ are at greater risk of death from GTN. In total, 14 patients had a FIGO score of $>16$.

Table 3 Univariate analysis of qualitative variables in gestational trophoblastic neoplasia patients with liver metastasis

\begin{tabular}{|c|c|c|c|c|c|c|c|}
\hline \multirow[t]{2}{*}{ Variables } & \multirow[t]{2}{*}{ Category } & \multicolumn{2}{|c|}{ Alive } & \multicolumn{2}{|c|}{ Deceased } & \multirow[t]{2}{*}{ Statistics } & \multirow[t]{2}{*}{$P$-value } \\
\hline & & $\mathbf{n}$ & $\%$ & $\mathbf{n}$ & $\%$ & & \\
\hline \multirow[t]{3}{*}{ Antecedent pregnancy } & Abortion & 8 & 36.36 & 6 & 33.33 & 8.622 & 0.013 \\
\hline & Mole & 9 & 40.91 & 1 & 5.56 & & \\
\hline & Term & 5 & 22.73 & 11 & 61.11 & & \\
\hline \multirow[t]{2}{*}{ Previous failed chemotherapy } & No & 15 & 68.18 & 5 & 27.78 & 6.465 & 0.011 \\
\hline & Yes & 7 & 31.82 & 13 & 72.22 & & \\
\hline \multirow[t]{2}{*}{ Brain metastasis } & No & 15 & 68.18 & 13 & 72.22 & 0.077 & 0.781 \\
\hline & Yes & 7 & 31.82 & 5 & 27.78 & & \\
\hline \multirow[t]{2}{*}{ Kidney metastasis } & No & 19 & 86.36 & 16 & 88.89 & 0.00 & 1.00 \\
\hline & Yes & 3 & 13.64 & 2 & 11.11 & & \\
\hline \multirow[t]{2}{*}{ Age, years } & $<40$ & 18 & 81.82 & 14 & 77.78 & 0.00 & 1.00 \\
\hline & $\geq 40$ & 4 & 18.18 & 4 & 22.22 & & \\
\hline \multirow[t]{2}{*}{ FIGO score } & $<12$ & 5 & 22.73 & 2 & 11.11 & 0.296 & 0.587 \\
\hline & $\geq 12$ & 17 & 77.27 & 16 & 88.89 & & \\
\hline \multirow[t]{2}{*}{ Surgery } & No & 12 & 54.5 & 14 & 77.8 & 2.35 & 0.125 \\
\hline & Yes & 10 & 45.5 & 4 & 22.2 & & \\
\hline \multirow[t]{3}{*}{ Number of metastatic sites (lung excluded) } & 1 & 11 & 50.0 & 9 & 50.0 & 1.626 & 0.444 \\
\hline & 2 & 9 & 40.9 & 5 & 27.8 & & \\
\hline & 3 & 2 & 9.1 & 4 & 22.2 & & \\
\hline
\end{tabular}

Abbreviation: FIGO, the International Federation of Gynecology and Obstetrics.

Table 4 Univariate analysis of quantitative variables

\begin{tabular}{|c|c|c|c|c|c|c|c|}
\hline Variables & Category & $\mathbf{n}$ & Mean \pm SD & Median & IQR & Range & $P$-value \\
\hline \multirow[t]{2}{*}{ Age, years } & Alive & 22 & $33.7 \pm 1.7$ & 33.0 & 8.0 & $21.0-54.0$ & 0.495 \\
\hline & Deceased & 18 & $31.8 \pm 1.7$ & 30.0 & 13.0 & $21.0-44.0$ & \\
\hline \multirow[t]{2}{*}{ FIGO score } & Alive & 22 & $14.0 \pm 0.7$ & 13.0 & 4.0 & $7.0-21.0$ & 0.002 \\
\hline & Deceased & 18 & $17.2 \pm 0.7$ & 17.0 & 4.0 & II.0-22.0 & \\
\hline \multirow[t]{2}{*}{ Gravidity } & Alive & 22 & $3.6 \pm 0.4$ & 4.0 & 3.0 & $1.0-6.0$ & 0.160 \\
\hline & Deceased & 18 & $2.8 \pm 0.4$ & 3.0 & 3.0 & $1.0-6.0$ & \\
\hline \multirow[t]{2}{*}{ Parity } & Alive & 22 & $1.1 \pm 0.2$ & 1.0 & 2.0 & $0.0-4.0$ & 0.760 \\
\hline & Deceased & 18 & $1.2 \pm 0.2$ & 1.0 & 1.0 & $0.0-3.0$ & \\
\hline \multirow[t]{2}{*}{$\beta$-hCG (log) } & Alive & 22 & $4.8 \pm 0.2$ & 5.0 & 1.3 & $1.6-6.5$ & 0.342 \\
\hline & Deceased & 18 & $4.5 \pm 0.2$ & 4.8 & 1.4 & $1.8-5.9$ & \\
\hline
\end{tabular}

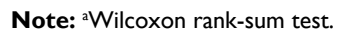

Abbreviations: $\beta$-hCG, beta-human chorionic gonadotropin; FIGO, the International Federation of Gynecology and Obstetrics; IQR, interquartile range. 


\section{Survival analyses}

The 5-year overall survival rate of the entire cohort, including the 3 patients who died during initial chemotherapy, was $55.7 \%$ (Figure 1A). The 5-year overall survival rate when excluding the 3 aforementioned patients was $60.2 \%$. Patients with a FIGO score of $>16$ had a significantly poorer survival than those with a score of $\leq 16(P<0.001)$ (Figure 1B).

\section{Discussion}

Liver metastases from GTN are rare. The incidence in our retrospective cohort of 2,067 patients was $1.9 \%$, which is consistent with that $(1.8 \%-2.0 \%)$ of previous reports from the Charing Cross Trophoblastic Disease Center (London, UK) ${ }^{3}$ and the John I. Brewer Trophoblastic Disease Center (Chicago, IL, USA). ${ }^{6}$ Due to the rarity of liver metastases from GTN, it is difficult to conduct prospective studies to investigate the optimal management and prognostic factors of GTN patients with liver metastases. Therefore, it is necessary to conduct large retrospective studies aimed at improving the outcomes of this subgroup of patients.

The CR rate in our study was $73.0 \%$, while the 5-year overall survival rate was $55.7 \%$ (or $60.2 \%$ excluding the 3 patients who died during initial chemotherapy). Compared with previous studies in which the survival rates for 1949-1964, 1965-1985, and 1985-1998 were 0.0\%, 23.3\%, and $37.5 \%,{ }^{8}$ respectively, the survival rate for $1999-2015$ was significantly higher than those of previous reports. This improvement may not be attributed to any obvious differences in the clinical characteristics of patients in different cohorts. Instead, it appears to be the result of introducing FAEV and EMA/CO regimens. Floxuridine-based combination che-

A

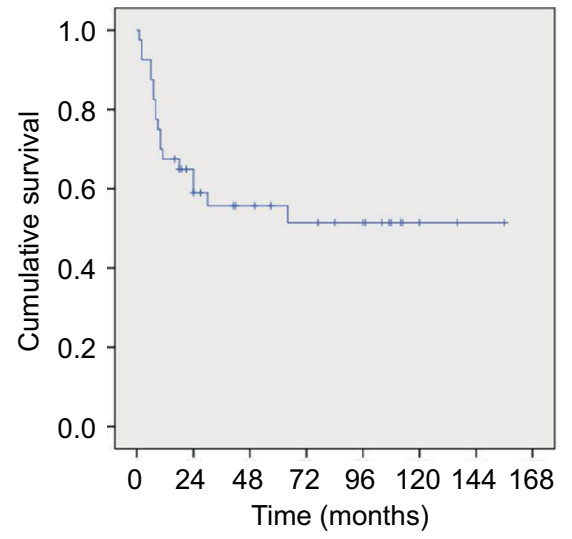

motherapy (FAEV) has been favored for the management of high-risk GTN patients for the past 3 decades in People's Republic of China. ${ }^{2,11,13}$ Single-agent mercaptopurine or chlormethine was the regimen of choice for GTN patients between 1945 and 1965, before the introduction of FAEV. ${ }^{9}$ Data from the Charing Cross Hospital suggest that the overall survival rate of patients with liver metastases improved from $27 \%$ to $48 \%$, owing to the introduction of EMA-CO and EP/ EMA during the past 3 decades. ${ }^{3}$ In addition, Barber et al from John I. Brewer Trophoblastic Disease Center reported that the introduction of EMA/CO improved the survival rate from $17 \%$ to $55 \%{ }^{6}$ The FAEV regimen was used as the primary chemotherapy in the majority of our patients. Our results indicate that a 5-year survival rate of $60.2 \%$ (excluding the 3 patients who died during initial chemotherapy) is similar to the disease-specific survival rate $(68.0 \%)$ reported at the Charing Cross $\mathrm{Hospital}^{3}$ after excluding 7 patients with early deaths. ${ }^{3}$ Hence, both FAEV and EMA/CO regimens appear to improve the survival of GTN patients with liver metastases. Owing to the small number of samples, we were unable to compare the efficacy and toxicity of FAEV and EMA/CO regimens in GTN patients with liver metastases.

In addition to chemotherapy, the removal of isolated pulmonary nodules and hysterectomy can improve the outcomes of stage IV GTN patients with isolated drug-resistant tumors. ${ }^{14}$ In our cohort, 14 patients underwent surgery (pelvic operations $[n=8]$ and thoracotomies $[n=6]$ ) to remove drug-resistant lesions. One patient underwent liver lesion resection. Similarly, Barber et al reported that 8 patients had undergone surgery (hysterectomy [ $\mathrm{n}=5]$, thoracotomy $[n=3]$, and craniotomy $[n=1])$ for the resection of residual

\section{B}

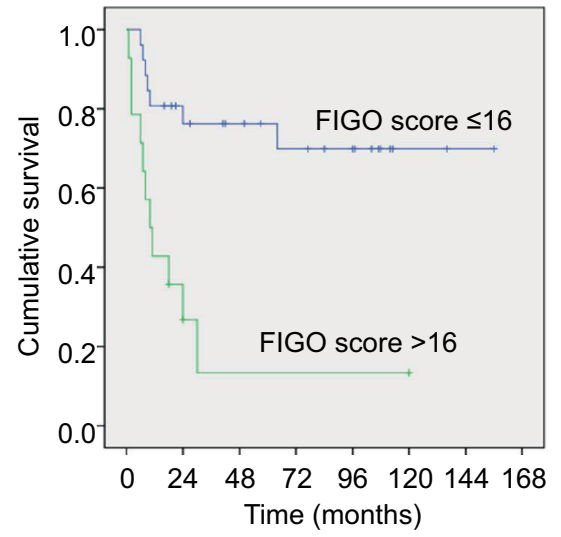

Figure I Kaplan-Meier survival curves.

Notes: Overall survival of $(\mathbf{A})$ gestational trophoblastic neoplasia patients with liver metastases $(\mathrm{n}=40)$ and $(\mathbf{B})$ patients with FIGO scores $\leq 16$ versus those with scores $>16$.

Abbreviation: FIGO, the International Federation of Gynecology and Obstetrics. 
disease. ${ }^{3}$ The surgical indications of GTN patients with liver metastasis are largely comparable to those of general GTN patients. Laparotomy or neurosurgery is required to stem the bleeding in organs such as the liver, gastrointestinal tract, and brain. Excision of isolated pulmonary nodules or hysterectomy for patients with an isolated drug-resistant lesion is also recommended. However, the role of liver lesion resection requires further investigation.

Owing to the rarity of liver metastases from GTN, the prognostic factors associated with this disease remain controversial. In the present study, multivariate analysis revealed that a history of failed multidrug chemotherapy was the only independent risk factor for predicting a poor survival. Therefore, patients diagnosed with GTN should be transferred to GTN-specialized centers, where they can receive appropriate management to prevent the development of drug resistance. Interestingly, we found that many common negative prognostic factors, such as older age, antecedent pregnancy, elevated serum $\beta$-hCG levels, and brain metastases, were not significantly associated with survival in GTN patients with liver metastases.

In 1997, Crawford et al summarized the experience of Charing Cross Hospital and demonstrated that a World Health Organization prognostic score of $>12$ was an independent significant factor for cause-specific survival in GTN patients with liver metastases. ${ }^{10}$ However, none of the variables tested were found to be prognostic factors in a more-recent 2012 study from the Charing Cross Hospital due to the small sample size. ${ }^{3}$ Our results show that patients with a FIGO score of $>16$ have poor survival outcomes. This suggests that GTN patients with liver metastases could be further divided into subgroups based on their FIGO scores. In such a case, stratified management could be provided, and more attention could be paid to those with FIGO scores $>16$.

Both brain and liver metastases were found to be poor prognostic factors in GTN patients in our previous studies, with 5-year overall survival rates of $71.1 \%$ and $55.7 \%$, respectively. ${ }^{2,4,11}$ Barber et $\mathrm{al}^{6}$ revealed that concomitant brain metastasis predicted shorter survival in patients with liver metastasis, although only 17 patients were included in their analysis. While brain metastasis was not associated with reduced survival according to the univariate and multivariate analyses, $41.7 \%$ of the patients $(n=5)$ died of the disease, which is a noteworthy statistic. The results of our analysis could also be attributed to the more aggressive nature and poorer disease course of GTN with liver metastases.

\section{Conclusion}

GTN with liver metastases is a very rare disease with a relatively poor prognosis. Patients with a history of failed multidrug chemotherapy and a FIGO score of $>16$ have poorer survival outcomes. Multidrug chemotherapy in combination with surgery, where necessary, is recommended as the primary treatment modality for GTN patients with liver metastases.

\section{Acknowledgments}

We wish to thank the medical record room staff for their assistance in retrieving the medical records of patients. This work was supported by a grant from the National Natural Science Foundation of China (grant number 81672586). The funding agency has no involvement with the design of the study or the collection, analysis, and interpretation of data, as well as writing of the manuscript.

\section{Disclosure}

The authors report no conflicts of interest in this work.

\section{References}

1. Ngan HY, Seckl MJ, Berkowitz RS, et al. Update on the diagnosis and management of gestational trophoblastic disease. Int J Gynaecol Obstet. 2015;131(suppl 2):S123-S126.

2. Xiao C, Yang J, Zhao J, et al. Management and prognosis of patients with brain metastasis from gestational trophoblastic neoplasia: a 24-year experience in Peking union medical college hospital. BMC Cancer. 2015; 15:318.

3. Ahamed E, Short D, North B, Savage PM, Seckl MJ. Survival of women with gestational trophoblastic neoplasia and liver metastases: is it improving? J Reprod Med. 2012;57(5-6):262-269.

4. Li J, Yang J, Liu P, et al. Clinical characteristics and prognosis of 272 postterm choriocarcinoma patients at Peking Union Medical College Hospital: a retrospective cohort study. BMC Cancer. 2016;16:347.

5. Bower M, Newlands ES, Holden L, et al. EMA/CO for high-risk gestational trophoblastic tumors: results from a cohort of 272 patients. $J$ Clin Oncol. 1997;15(7):2636-2643.

6. Barber EL, Schink JC, Lurain JR. Hepatic metastasis in gestational trophoblastic neoplasia: patient characteristics, prognostic factors, and outcomes. J Reprod Med. 2014;59(5-6):199-203.

7. Yang J, Xiang Y, Wan X, Feng F, Ren T. Analysis of the prognosis and related factors for patients with stage IV gestational trophoblastic neoplasia. Int J Gynecol Cancer. 2014;24(3):594-599.

8. Xiang Y, Yang X, Yang N, Chen C, Cao T, Song H. Diagnosis and management of gestational trophoblastic tumor with liver metastases. Zhongguo Yi Xue Ke Xue Yuan Xue Bao. 2000;22(1):41-43.

9. Wang YE, Song HZ. Liver metastasis of choriocarcinoma. Report of 44 patients. Chin Med J. 1988;101(9):637-642.

10. Crawford RA, Newlands E, Rustin GJ, Holden L, A'Hern R, Bagshawe KD. Gestational trophoblastic disease with liver metastases: the Charing Cross experience. Br J Obstet Gynaecol. 1997;104(1):105-109.

11. Kong Y, Yang J, Jiang F, et al. Clinical characteristics and prognosis of ultra high-risk gestational trophoblastic neoplasia patients: a retrospective cohort study. Gynecol Oncol. 2017;146(1):81-86.

12. Ngan HY. The practicability of FIGO 2000 staging for gestational trophoblastic neoplasia. Int J Gynecol Cancer. 2004;14(2):202-205. 
13. Yang J, Xiang Y, Wan X, Feng F, Ren T. Primary treatment of stage IV gestational trophoblastic neoplasia with floxuridine, dactinomycin, etoposide and vincristine (FAEV): a report based on our 10-year clinical experiences. Gynecol Oncol. 2016;143(1):68-72.
14. Fulop V, Szigetvari I, Szepesi J, Vegh G, Zsirai L, Berkowitz RS. The role of surgery in the management of gestational trophoblastic neoplasia the Hungarian experience. J Reprod Med. 2016;61(5-6):197-204.
Cancer Management and Research

\section{Publish your work in this journal}

Cancer Management and Research is an international, peer-reviewed open access journal focusing on cancer research and the optimal use of preventative and integrated treatment interventions to achieve improved outcomes, enhanced survival and quality of life for the cancer patient The manuscript management system is completely online and includes a very quick and fair peer-review system, which is all easy to use. Visit $\mathrm{http}: / / \mathrm{www}$. dovepress.com/testimonials.php to read real quotes from published authors. 